\title{
Characterizing Alnico Alloy by Correlative STEM-EDS Tomography and Atom Probe Tomography
}

Wei Guo, ${ }^{1}$ Brian Sneed, ${ }^{1}$ Lin Zhou, ${ }^{2}$ Wei Tang, ${ }^{2}$ Matthew. J. Kramer, ${ }^{2}$ David A. Cullen, ${ }^{3}$ and Jonathan D. Poplawsky ${ }^{1}$

1. Center for Nanophase Materials Sciences, Oak Ridge National Laboratory, Oak Ridge, TN, USA.

2. Ames Lab, Ames, IA, USA.

3. Materials Science and Technology Division, Oak Ridge National Laboratory, Oak Ridge, TN, USA.

Alnico alloys are a family of hard magnetic functional alloys that are composed of $\mathrm{Al}, \mathrm{Ni}, \mathrm{Co}$, and $\mathrm{Fe}$. The magnetic properties of alnico alloys are highly linked with nanostructure formation during a rapid quenching, phase decomposition process, in which a single $\alpha$-phase at $\sim 1250^{\circ} \mathrm{C}$ (BCC structure) is transformed into a hard magnetic FeCo-rich $\alpha_{1}$-phase (B2 structure) and a non-magnetic NiAl-rich phase $\alpha_{2}$-phase ( $\mathrm{L}_{2}$ structure). Previous experiments and theoretical predictions indicate that improving alnico requires subtle changes in chemistry and processing to reduce the diameter of the $\alpha_{1}$-phase while maintaining its volume fraction [1]. As the size of the decomposed phase scales from $\sim 1 \mathrm{~nm}$ to tens of $\mathrm{nms}$, it becomes challenging to identify the exact morphology, volume fraction, and atomic-scale landscape of phase chemistry and structure. Advanced microscopy can provide improved understanding of the phase decomposition of such alloys and the corresponding property optimization.

Linking 3-D tomography perspectives across multiple length-scales and characterization techniques is progressively being utilized across a wide range of materials science to advance understanding. Among the various tomography methods, scanning transmission electron microscopy (STEM)-based tomography capabilities have improved over the past decade [2] and can be used to construct information in $3 \mathrm{D}$ for a probed volume up to $300 \times 300 \times 500 \mathrm{~nm}^{3}$ by reconstructing a series of $2-\mathrm{D}$ projected images acquired at various tilt angles followed by $3 \mathrm{D}$ data processing. The spatial resolution of this technique ranges from 1 to $10 \mathrm{~nm}$. Atom probe tomography (APT) is another tool that provides sub-nm spatial resolution and chemical sensitivity of $10 \mathrm{ppm}$ for all elements, which complements the scale and chemical sensitivity not covered by STEM-based tomography. However, the region of analysis for APT is normally confined to a small volume, which may not reflect the overall microstructural landscape when the unit of structure is beyond $50 \mathrm{~nm}$. Also, APT data are subject to image distortions due to field evaporation aberrations that are sometimes impossible to correct. STEM-imaging and energy dispersive X-ray spectroscopy (EDS) tomography experiments conducted prior to APT analysis is important to clearly understand the material's nanostructure with higher accuracy than APT alone.

Correlative STEM-EDS/APT was conducted for an alnico 8 alloy to investigate phase separation from the sub- $\mu \mathrm{m}$ to the sub-nm scale. Figure 1 shows the correlative STEM/APT data of the alnico 8 alloy annealed at $840^{\circ} \mathrm{C}$ for $90 \mathrm{~s}$, followed by water quenching to room-temperature. The high angle annular dark field (HAADF)-STEM image (Fig. 1a) shows atomic number (Z)-contrast that clearly differentiates the phase morphology in the APT tip; e.g., the Fe-enriched $\alpha_{1}$-phase appears brighter because of the higher average $\mathrm{Z}$ than the $\alpha_{2}$-phase. The yellow dashed line shows the area of the STEM tomography reconstruction (Fig. 1b), for a collected volume of $1.46 \times 10^{6} \mathrm{~nm}^{3}$. The red dashed lines mark the $2.3 \times 10^{5}$ $\mathrm{nm}^{3}$ volume for APT data acquisition (Fig. 1c). For STEM-EDS tomography (Fig. 1b), only Fe and Ni are shown for clarity. The $\alpha_{1}$-phase consists of a mixture of cube-shaped tiles with a $\sim 35 \mathrm{~nm}$ average length, and elongated rods, which developed by spinodal decomposition. The 30at.\% Fe 
isoconcentration surface in Fig. 1c clearly show multiple $\alpha_{1} / \alpha_{2}$ interfaces. Such isosurfaces not only confirm the appearance of the uncontrolled elongated nanostructure of $\alpha_{1}$-phase at the bottom of the APT volume, but also indicate the existence of Fe-enriched inclusions embedded inside the $\alpha_{2}$-phases.

Two regions of interest were further probed for composition analysis. The first sampling volume $\left(10 \times 10 \times 17 \mathrm{~nm}^{3}\right)$ was selected across an $\alpha_{1} / \alpha_{2}$ boundary (Fig. 1c). As a consequence of the APT needle orientation close to the (100) pole, the (100) lattice planes can be resolved. The 1D concentration analysis indicates a composition of 4.6 at.\% Al, 1.1 at.\% Ti, 36.7 at.\% Co, 1.2 at.\% Cu, 51.6 at.\% Fe, and 4.6 at. $\% \mathrm{Ni}$ for the $\alpha_{1}$-phase, and 22.1 at.\% $\mathrm{Al}, 14.6$ at.\% Ti, 33.2 at.\%Co, 1.8 at.\% $\mathrm{Cu}, 13.6$ at.\% Fe, and 14.6 at. $\% \mathrm{Ni}$ for the $\alpha_{2}$-phase. The second probed area was extracted from the region where a Ferich region was embedded in the $\alpha_{2}$ phase (Fig. 1c). The proximity histogram analysis shows the Fe-rich region has a composition of 48.7 at.\% $\mathrm{Fe}, 37.5 . \%$ at.\% $\mathrm{Co}, 4.8$ at.\% $\mathrm{Al}, 0.8$ at.\% Ti, and 0.7 at.\% $\mathrm{Cu}$, matching the measured $\alpha_{1}$-phase composition.

The complementary 3D results from both techniques suggest that the phase decomposition of the alnico alloy is comprised of both spinodal decomposition and a nucleation and growth process at $840{ }^{\circ} \mathrm{C}$ (if no external magnetic field is applied) which facilitates a better understanding of its magnetic properties. The demonstrated experimental approach will provide a powerful tool to investigate the structure and chemistry of a wide range of other nanomaterials and nanodevices. [3]

References:

[1] L. Zhou et al., Acta Mater. 74 (2014) p. 224.

[2] P.A. Midgley, R.E. Dunin-Borkowski, Nat. Mater. 8 (2009) p. 271.

[3] Research supported by U.S. DOE, office of Energy Efficiency and Renewable Energy (EERE), under its Vehicle Technologies Program, through the Ames Laboratory, Iowa State University under contract DE-AC02-07CH11358. The STEM instrumentation is provided by the U.S. DOE Office of Nuclear Energy, Fuel Cycle R\&D Program, and the Nuclear Science User Facilities. APT research was conducted at ORNL's Center for Nanophase Materials Sciences (CNMS), which is a DOE Office of Science User Facility.

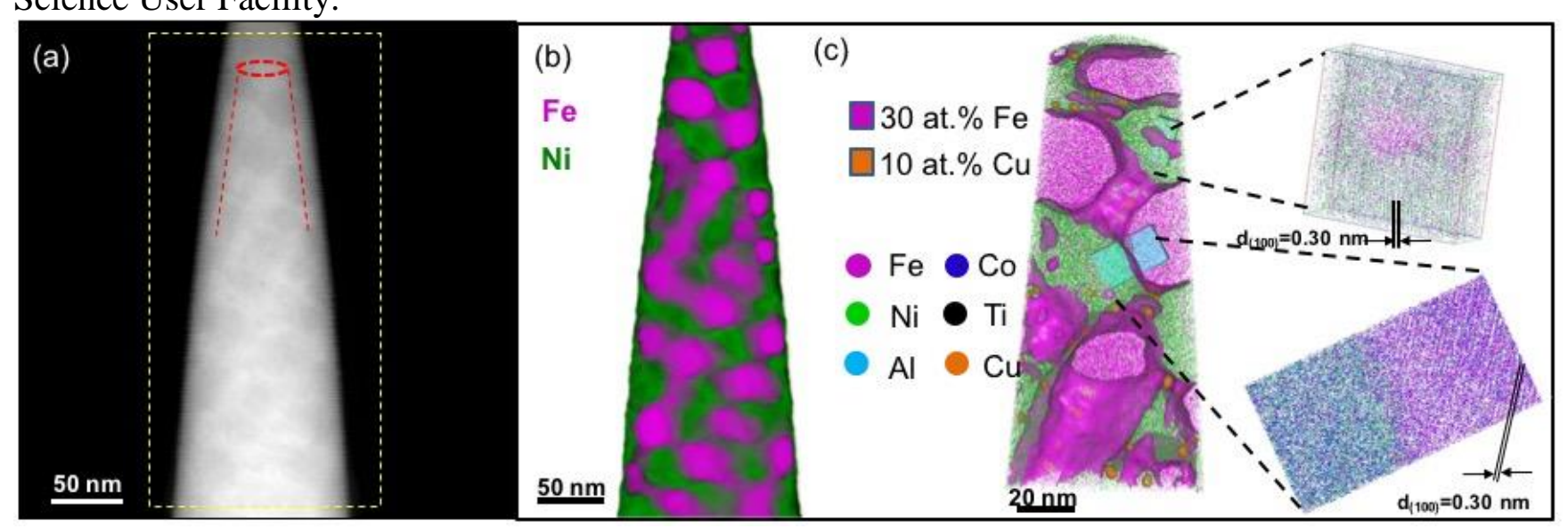

Figure 1. Combined STEM-EDS/APT tomography characterization of alnico 8 alloy. (a) HAADFSTEM image shows cube-morphology of $\alpha_{1^{-}} \mathrm{a} \square \square \square \alpha_{2}-\square \square \square \square \square \mathrm{s}$. (b) 3D reconstructed volume from STEM-EDS tomography - Fe and Ni components are shown for clarity. (c) APT reconstructed volume 30 at.\% isoconcentration surfaces identify the $\alpha_{1} / \alpha_{2} \square \square \square \square \square$ face and 30 at.\% $\mathrm{Cu}$ isosurfaces show $\mathrm{Cu}$ precipitates. The magnified volumes clearly show (100) lattice planes for both $\alpha_{1}-\mathrm{a} \square \square \alpha_{2}-\mathrm{p} \square \square \square \square \square \square$ 